\title{
Erdheim-Chester disease: two cases of orbital involvement
}

\begin{abstract}
Erdheim-Chester disease (ECD) is an increasingly recognised form of fibroinflammatory process characterised by xanthomatous histiocytes containing large amounts of ingested lipid, plasma cells, macrophages and Touton-type giant cells. Ophthalmic involvement in ECD has been reported in only 22 cases. We describe two patients, one presenting with diabetes insipidus and subsequently developing orbital pseudotumours and retroperitoneal fibrosis, the other presenting with exophthalmos and diplopia. The first patient was treated with cladribine and subsequently developed sudden onset of bilateral blindness while the second required radiation therapy for the retro-orbital process and developed radiation retinopathy. These cases typify the variable presentation and course in patients with ECD.
\end{abstract}

\section{T.G. Sheidow \\ D.A. Nicolle \\ J.G. Heathcote \\ Department of \\ Ophthalmology University of Western \\ Ontario \\ London, Canada \\ J.G. Heathcote \\ Department of Pathology University of Western \\ Ontario \\ London, Canada \\ Tom Sheidow \\ Department of \\ Ophthalmology \\ University of Western \\ Ontario \\ London Health Sciences \\ Centre \\ 750 Commissioners Road E. \\ London, Ontario \\ Canada N6A 4G5 \\ e-mail: \\ tgsheido@julian.uwo.ca}

Proprietary interest: None

Received: 27 September 1999

Accepted in revised form: 25 February 2000
Key words Cladribine (2-CDA), Complications, Erdheim-Chester disease, Ocular manifestations, Orbital pseudotumour

Erdheim-Chester disease (ECD) is an increasingly recognised form of histiocytosis characterised by a fibro-inflammatory process involving xanthomatous histiocytes containing large amounts of ingested lipid, plasma cells, macrophages and Touton-type giant cells. The first case of ECD was reported in 1930 by Chester. ${ }^{1}$ An extensive literature review in 1996 by Veyssier-Belot $e t$ al. ${ }^{2}$ found only 59 reported cases of ECD of which 16 had orbital involvement. Subsequently, 7 further cases $^{3-8}$ with orbital involvement have been reported. We present two cases of ECD involving the orbit with very different presentations and outcomes.

\section{Case reports}

Case 1

A 57-year-old man with a history of polyuria and polydipsia was referred to the endocrine service in 1995 where a diagnosis of diabetes insipidus of both neurogenic and nephrogenic origin was made. Magnetic resonance imaging
TOM G. SHEIDOW, DAVID A. NICOLLE, J. GODFREY HEATHCOTE
(MRI) at the time of diagnosis showed minimal enlargement of the pituitary infundibulum; no orbital lesions were noted. His systemic condition remained stable for $1 \frac{1 / 2}{2}$ years at which time a routine chest radiograph showed cardiomegaly and prominent pulmonary vasculature. A moderate pericardial effusion was found and treated by the creation of a pericardial window on two separate occasions. Pathological examination of the pericardial tissue revealed fibrosis and active chronic inflammation.

In February 1997, bilateral periorbital skin changes prompted consultation with a dermatologist and the clinical diagnosis of xanthelasma was confirmed by biopsy. The ophthalmology service was also consulted because of symptoms of swelling around both eyes, soreness and photophobia.

Examination revealed visual acuities of 20/20 in both eyes with full extraocular movements and mild lid lag. Diffuse xanthelasmata in the periorbital skin were noted and there was a bilateral superficial punctate keratopathy. The patient was treated for dry eyes and followed by the ophthalmology service at monthly intervals.

Two months later the patient was admitted to hospital with hyponatraemia and confusion secondary to the use of DDAVP (desmopressin). At that time slight, bilateral proptosis was noted with mild disc swelling but a computed tomography (CT) scan of the head was reported as normal. A subsequent deep vein thrombosis was accompanied by bilateral pleural effusions, suggestive of pulmonary thromboembolism. A $\mathrm{CT}$ scan of the abdomen at this time revealed a large area of retroperitoneal fibrosis.

In July 1997, radiographs of the femurs and tibias showed symmetrical osteosclerosis in the diaphyseal and metaphyseal regions. The combination of diabetes insipidus, pericardial effusions, retroperitoneal fibrosis and long bone changes raised the possibility of ECD. At the same time the neuro-ophthalmology service was consulted because of continuing difficulties with periorbital swelling, ocular irritation, tearing and near-vision. Examination at that time revealed acuities of 20/50 in the right eye and $20 / 200$ in the left, with pinhole correction to $20 / 30$ and 20/60 respectively. The extraocular 


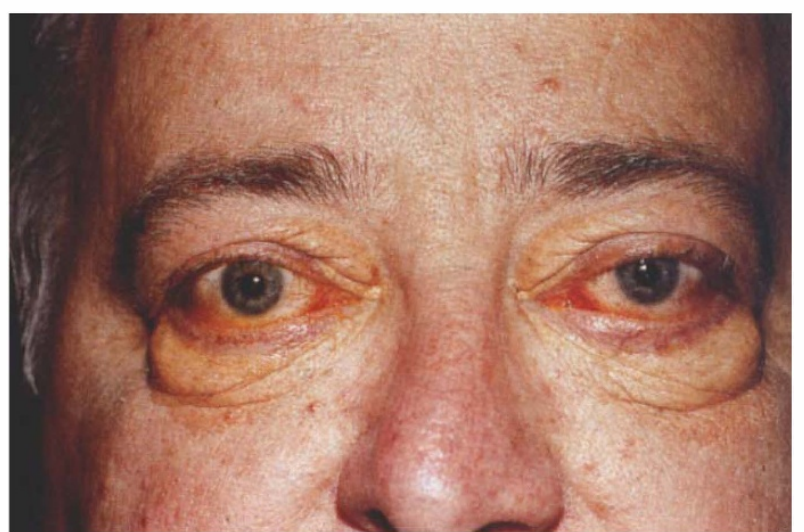

(a)

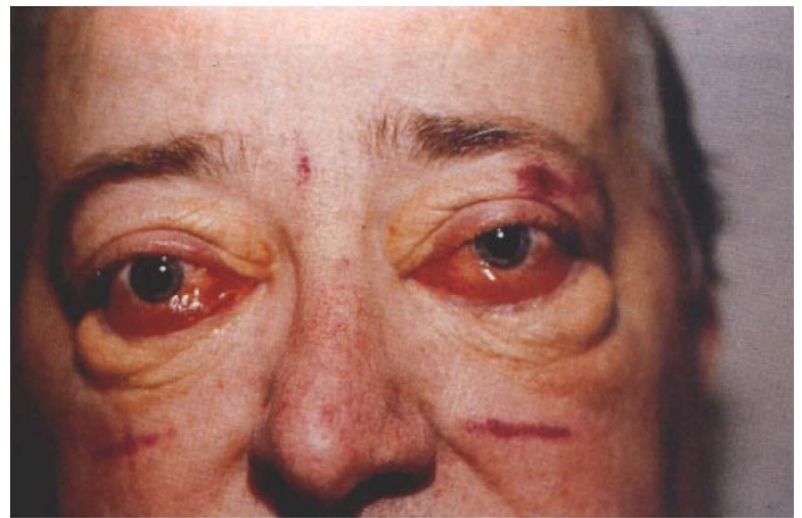

(b)

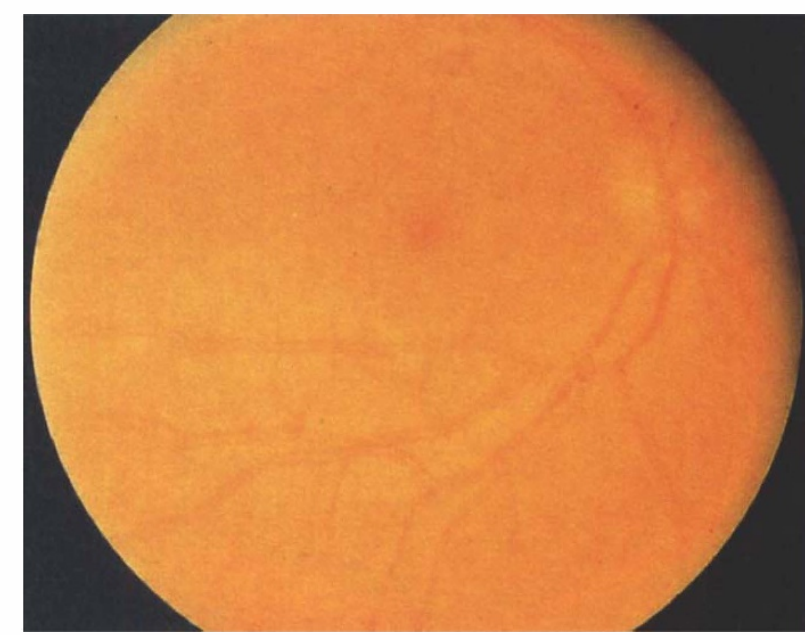

(c)

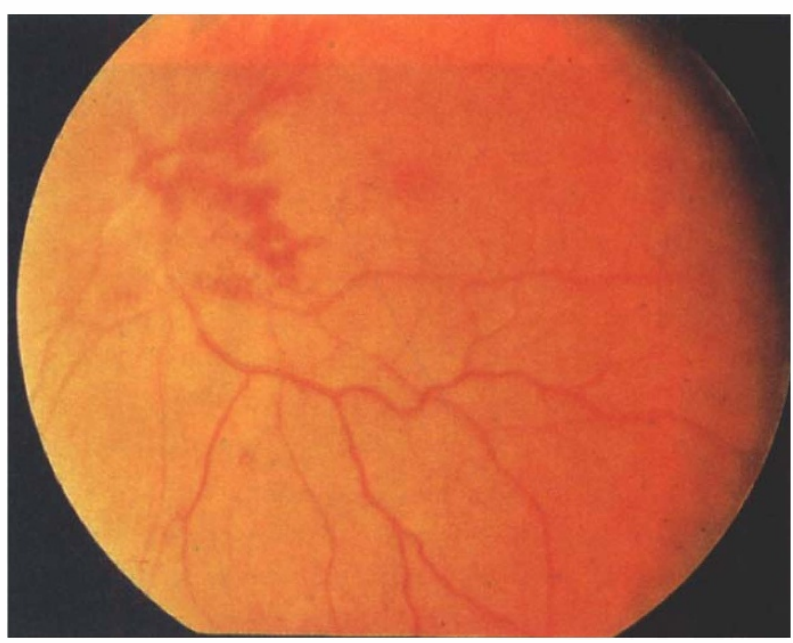

(d)

Fig. 1(a)-(d). Case 1. External photograph of bilateral xanthelasmata, conjunctival chemosis and proptosis (a). External photograph of case 1 after a sudden decrease in visual acuity $(b)$, and corresponding disc photographs $(c, d)$ depicting swelling and haemorrhage within the optic disc bilaterally. (contd.)

movements showed horizontal and vertical gaze restrictions with ptosis of the left eye and marked proptosis (26 and $27 \mathrm{~mm}$ ) (Fig. 1a). Conjunctival injection was present but the intraocular pressures were normal and fundus examination revealed only mild nasal blurring of the discs. Goldmann fields were normal. Urgent CT scans of the orbits showed bilateral proptosis with substantial intraconal soft tissue masses consistent with orbital pseudotumours (Fig. 2).

The patient was started on prednisone $100 \mathrm{mg}$ o.d. and appeared to remain stable from an ocular perspective. In September 1997, approximately 1 month later, the patient began a course of chemotherapy with three cycles of cladribine (2-CDA), each consisting of 11.5 $\mathrm{mg}$ daily for 5 days. Following the completion of the first cycle the patient noticed an increase in the periorbital swelling and decreasing vision (Fig. 1b). Examination showed an increase in the degree of chemosis and continued restriction of ocular movement. Hertel measurements of $28 \mathrm{~mm}$ in the right eye and $30 \mathrm{~mm}$ in the left were obtained. Both optic discs remained normal. Radiation therapy was requested on an urgent basis but, before this could be given, the patient was admitted to the hospital 3 days later with a sudden onset of bilateral blindness, noted upon awakening. Visual acuity was no light perception in either eye with bilateral unreactive pupils. Initially neither disc showed evidence of swelling or pallor suggestive of a compressive effect of the pseudotumour. Intravenous solumedrol ( $1 \mathrm{~g} /$ day) and radiation therapy ( $2500 \mathrm{cGy}$ ) in 10 doses was given for 5 days without benefit. Repeat CT scans of the orbits showed no change in the retrobulbar masses. However, within 1 week, swelling and haemorrhage was noted within the optic disc bilaterally (Figs. 1c, d).

\section{Histopathological findings}

The eyelid and pericardial biopsies were reviewed. In the dermis of the eyelid there was a patchy infiltrate of foamy macrophages of differing sizes. A few foamy cells were large with multiple nuclei and had the appearance of Touton giant cells (Fig. 3). The well-circumscribed collections of foamy macrophages typical of xanthelasma were not seen. The pericardial tissue contained a mixed 


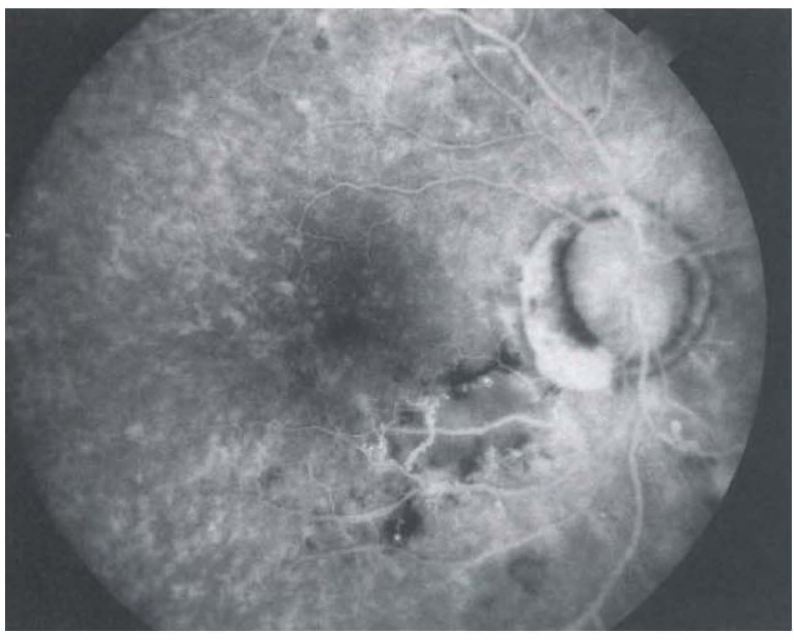

(e)

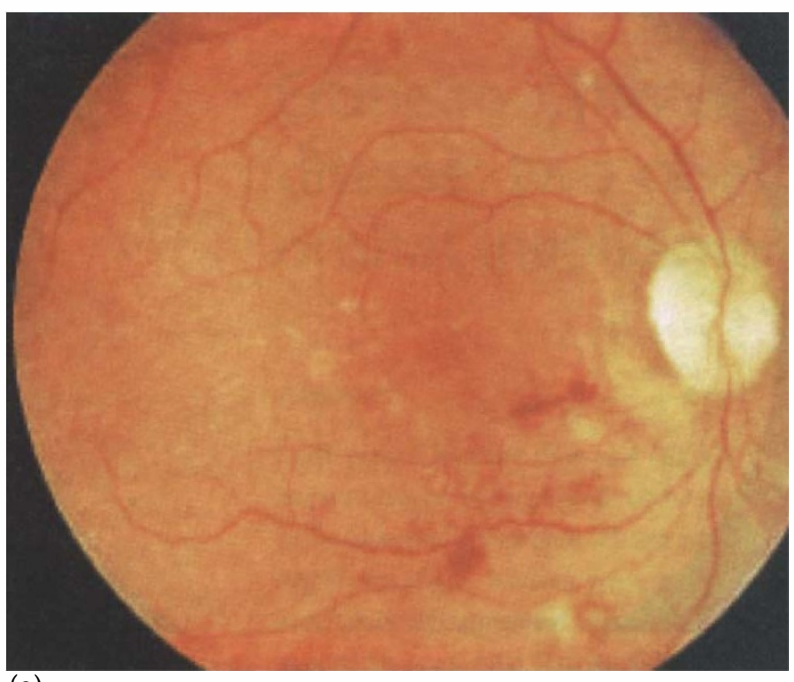

(g)

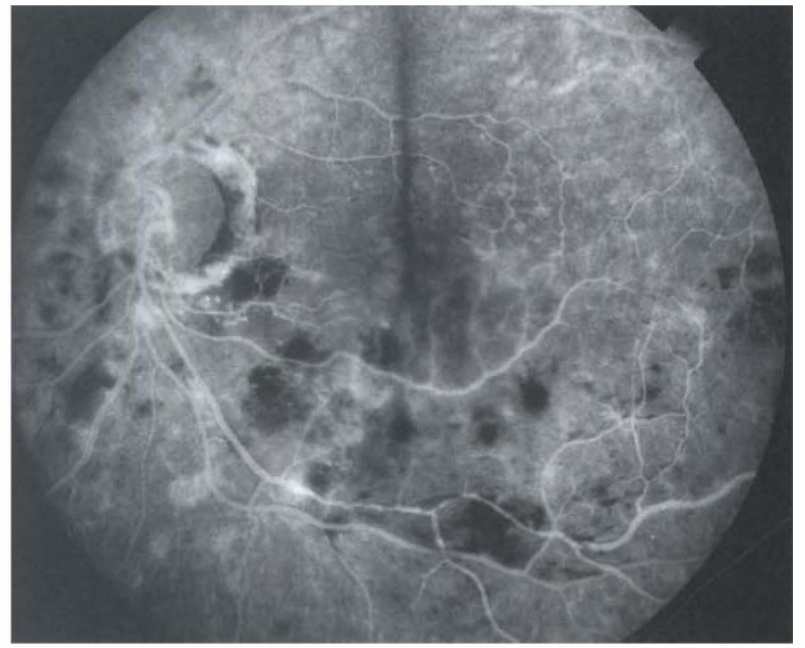

(f)

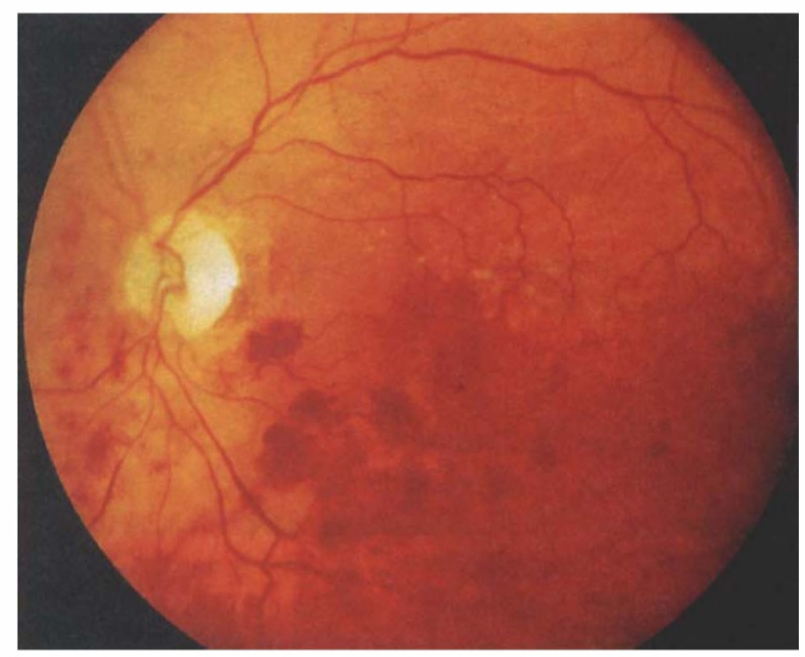

(h)

Fig. 1(e)-(h). Case 2. Disc photographs of patient 2 at the time of diagnosis of radiation retinopathy (e, f). Note the multiple intraretinal haemorrhages and nerve fibre layer infarcts with evidence of multiple venous anomalies. Bilateral macular thickening is also present. Intravenous fluorescein angiography $(g, h)$ showed evidence of non-perfusion in the macula of both eyes. The parafoveal arcade is markedly disrupted in the left eye (h). Neovascularisation is present but no evidence of vascular sheathing is seen.

inflammatory cell infiltrate. Occasional histiocytes had foamy cytoplasm with more than one nucleus but classical Touton giant cells were not identified.

\section{Case 2}

A 46-year-old man presented in early 1994 to the neuroophthalmology service with a 1 month history of blurred vision and occasional diplopia on lateral gaze. He had no other ocular symptoms and his systemic medical history revealed only hypertension that was under good control. Visual acuities were 20/20 in the right eye and 20/60 in the left, with normal pupils. Examination revealed restriction of gaze in the left eye with diplopia in all positions and exophthalmometry indicated proptosis of 17 and $21 \mathrm{~mm}$ in the right and left eyes respectively. On slit-lamp examination there was mild chemosis in both eyes and intraocular pressures of $26 \mathrm{mmHg}$ in the right eye and $27 \mathrm{mmHg}$ in the left. Fundus examination revealed mild pallor of the discs but no oedema. Fine chorioretinal folds could be seen inferiorly adjacent to the disc in the left eye.

The initial impression was that of possible orbital lymphoma and an MRI scan was obtained that revealed the presence of diffuse infiltration of both orbits, more marked on the left (Fig. 4). A conjunctival biopsy showed a mixed inflammatory infiltrate and, with a likely diagnosis of inflammatory pseudotumour, the patient was started on prednisone $80 \mathrm{mg}$ daily. The patient was followed closely, with both subjective improvement and an increase in visual acuity in the left eye but little change in the degree of chemosis or proptosis.

Repeat MRI 3 months later suggested enlargement of the orbital infiltrates, prompting a biopsy of the left orbit. This was interpreted as a sclerosing pseudotumour and, in light of the association between pseudotumour and retroperitoneal fibrosis, consultations with internal medicine and urology were obtained. A CT scan of the abdomen and a pyelogram confirmed the presence of 


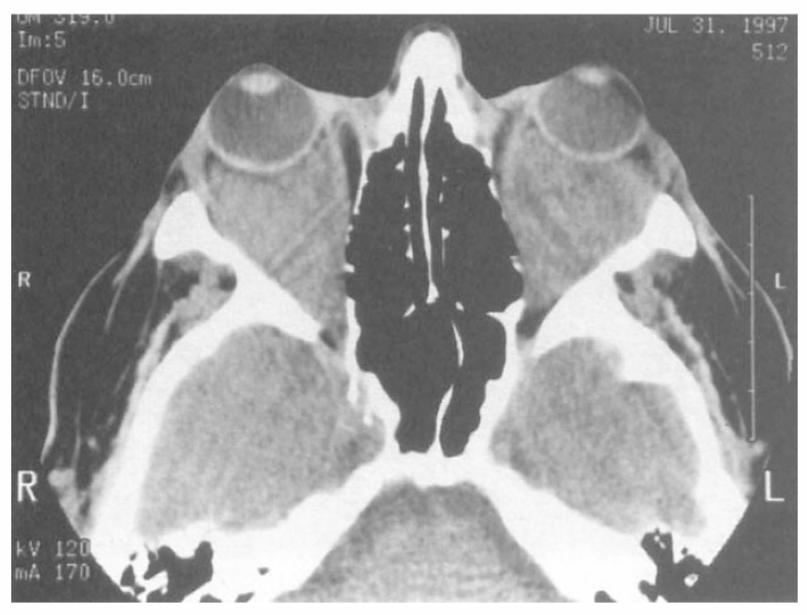

Fig. 2. Case 1. CT scan (axial) showing extensive bilateral orbital pseudotumours.

retroperitoneal fibrosis and ureteral stenosis.

Radiotherapy (4000 cGy) was given to the orbit and over the next 6 months the patients' ocular condition remained stable. However, the urological problems and hypertension related to the retroperitoneal fibrosis remained problematic and chemotherapy with azathioprine, initially $75 \mathrm{mg}$ o.d. then increased to $75 \mathrm{mg}$ b.i.d., was introduced. While on chemotherapy there was a gradual reduction in the restriction of the ocular movement. After 6 months of chemotherapy MRI showed no evidence of further progression in the size of the orbital pseudotumours. Azathioprine was continued and the prednisone gradually tapered.

In September 1995, the patient was seen in follow-up and complained of left hip and back pain. Routine radiographs showed patchy medullary sclerosis and cortical thickening of the left proximal humerus, the distal femur bilaterally and the left proximal tibia. A bone scan indicated increased uptake of isotope in these areas and a tibial biopsy revealed thickening of the cortical bone and foamy macrophages infiltrating the marrow spaces. A clinical diagnosis of ECD was made.

The patient remained stable on azathioprine and prednisone was tapered until December 1996, at which time diplopia returned with increased chemosis and

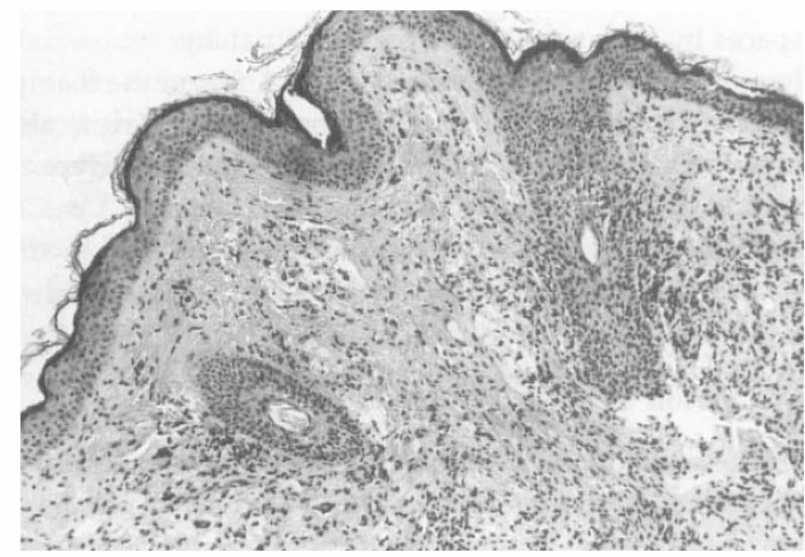

(a)

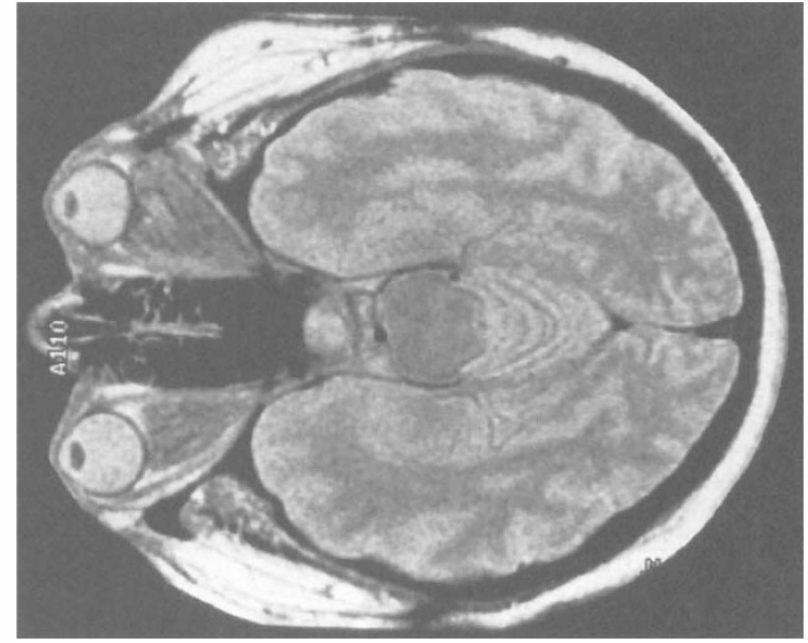

Fig. 4. Case 2. MRI scan of the orbital pseudotumours at the time of diagnosis.

proptosis. Hertel measurements of $23 \mathrm{~mm}$ in the right eye and $25 \mathrm{~mm}$ in the left were recorded. Visual acuity had decreased to 20/25 in the right eye and 20/100 in the left, and repeat MRI showed some increase in the size of the orbital infiltrates. Radiation treatment (2000 cGy in 10 treatments) and prednisone $30 \mathrm{mg}$ daily were re-initiated in January 1997. Again, following radiotherapy, the ocular condition stabilised and visual acuity returned to $20 / 40$ in the left eye.

Subsequently, the patient had progressive problems related to the increasing size of the retroperitoneal mass and its secondary effects on the urinary tract, with bilateral hydronephrosis requiring multiple stent procedures, nephrolithiasis, and finally renal failure on the right side. Echocardiography revealed diffuse global cardiac dysfunction but no evidence of pericardial disease. He gained significant amounts of weight secondary to the prednisone treatment and became diabetic.

In March 1999, following almost 2 years of stability in his ocular condition, the patient presented with a complaint of poor visual acuity of 10 days duration. The initial impression was of compression of the optic nerves and oral prednisone $50 \mathrm{mg}$ o.d. was initiated. An initial

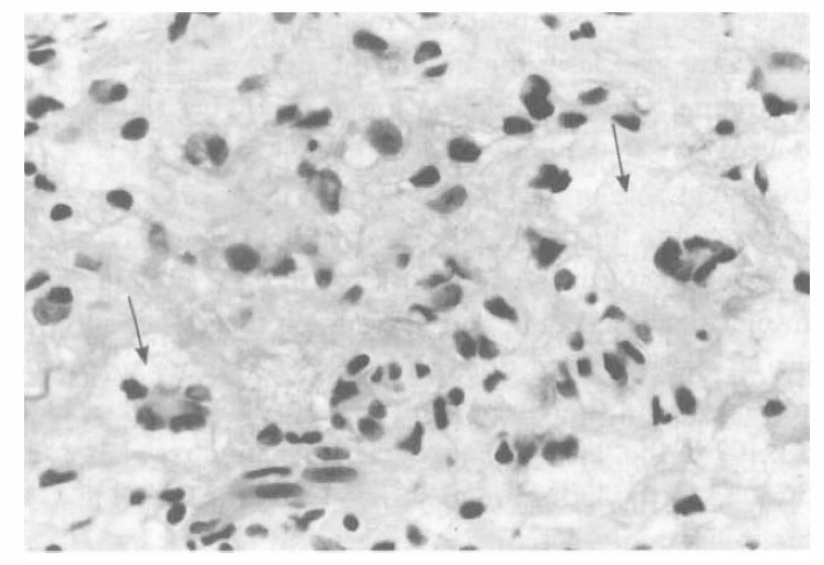

(b)

Fig. 3. Case 1. Biopsy of eyelid showing inflammatory infiltrate within the dermis (a). Scattered foamy macrophages are present and some are multinucleated and have the appearance of Touton giant cells (arrows, b). Haematoxylin and eosin; $(a) \times 100,(b) \times 800$. 


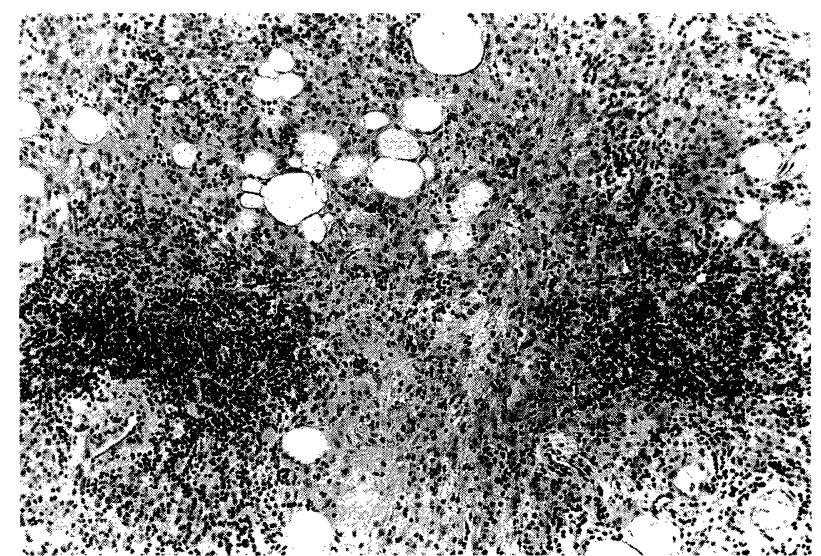

(a)

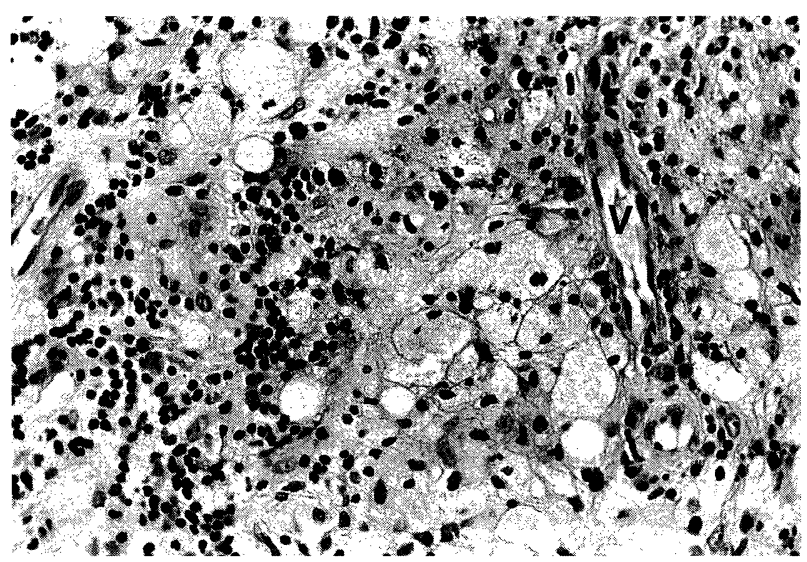

(b)

Fig. 5. Case 2. Orbital biopsy showing replacement of the orbital fat by fibro-in flammatory tissue (a). A small collection of foamy macrophages is seen adjacent to a blood vessel $(v)$ within the inflammatory infiltrate $(b)$. Haematoxylin and eosin; $(a) \times 125,(b) \times 400$.

increase and subsequent decrease in vision occurred and vitreoretinal consultation was obtained. On examination, visual acuity was $20 / 40$ in the right eye and 20/70 in the left. No afferent pupillary defect was present. Moderate chemosis and extraocular motility restriction were present with normal anterior segment examinations in both eyes. Dilated fundus examination revealed optic atrophy bilaterally with multiple small intraretinal haemorrhages in the inferior aspect of the left macula and nerve fibre layer infarcts (Fig. 1 e, f). Thickening of the retina extended from this area through the fovea. Nasal to the disc there was a notable area along the inferonasal vein that appeared to have been previously occluded and recanalised. In the left eye, more extensive thickening of the retina with greater numbers of nerve fibre layer infarcts and haemorrhages were observed inferiorly. No change in the calibre of the vessels was noted, suggestive of retrobulbar compression.

A working diagnosis of multiple branch retinal vein occlusions, secondary to radiation retinopathy, was made. An intravenous fluorescein angiogram revealed areas of non-perfusion inferiorly in both eyes and some neovascularisation (Fig. $1 \mathrm{~g}$, h). The parafoveal arcade was markedly disrupted in the right eye. Arteriovenous transit times were normal and no evidence of vasculitis was present. The patient was maintained on prednisone, and focal grid laser therapy to the right macula and panretinal photocoagulation initiated. Owing to the degree of ischaemia in the left macula, no direct treatment was undertaken: vision in this eye slowly improved as the degree of macular thickening decreased.

Tapering of the steroids resulted in a definite increase in macular thickening and a decline in visual acuity to $20 / 80$. An increased dose of steroids produced no response, and the visual acuity deteriorated further to $20 / 400$ in the left eye. Focal grid laser therapy was applied to the left eye and the oral prednisone dose tapered rapidly. Despite this, vision in the left eye remained at 20/400. A decrease in acuity in the right eye to $20 / 400$ occurred secondary to a dense premacular haemorrhage but resolved without intervention.

\section{Histopathology}

The conjunctival, orbital and tibial biopsies were reviewed. Within the conjunctival epithelium, which was focally parakeratotic, there were scattered neutrophils. The substantia propria contained a mixed inflammatory cell infiltrate and there was marked lymphangiectasia. The lymphoplasmacytic component was judged to be polyclonal on the basis of immunohistochemistry and neither foamy macrophages nor Touton giant cells were identified.

Review of the orbital biopsy from April 1994 revealed extensive replacement of orbital fat with fibrous tissue containing a diffuse mixed inflammatory infiltrate that included eosinophils and neutrophils (Fig. 5). There were numerous epithelioid histiocytes but no discrete granulomata. A high proportion of the histiocytes present had foamy cytoplasm and a few had more than one nucleus. However, classical Touton giant cells were not seen. Blood vessels with prominent endothelial cells and thickened walls were abundant. Lymphocytes were seen within the thickened vascular walls but there was no vasculitis. Histochemical stains for micro-organisms were negative and immunohistochemistry indicated a mixed infiltrate of $\mathrm{T}$ and $\mathrm{B}$ lymphocytes.

The core biopsy of the tibia revealed thickening of the bony trabeculae and diffuse replacement of the marrow spaces by foamy macrophages and a patchy lymphoplasmacytic infiltrate (Fig. 6). A few of the foamy cells had multiple nuclei but Touton giant cells were not identified. Occasional multinucleated osteoclasts were seen in a paratrabecular location.

The orbital and tibial biopsies were considered consistent with the clinical diagnosis of ECD.

\section{Discussion}

ECD is an idiopathic systemic disorder characterised by a sclerosing xanthogranulomatous process involving the long bones, heart, lungs and retroperitoneum. The disease is typically progressive in nature. A 1994 review by Veyssier-Belot et al. ${ }^{2}$ found only 59 cases reported in the literature. Orbital involvement in this disease was 


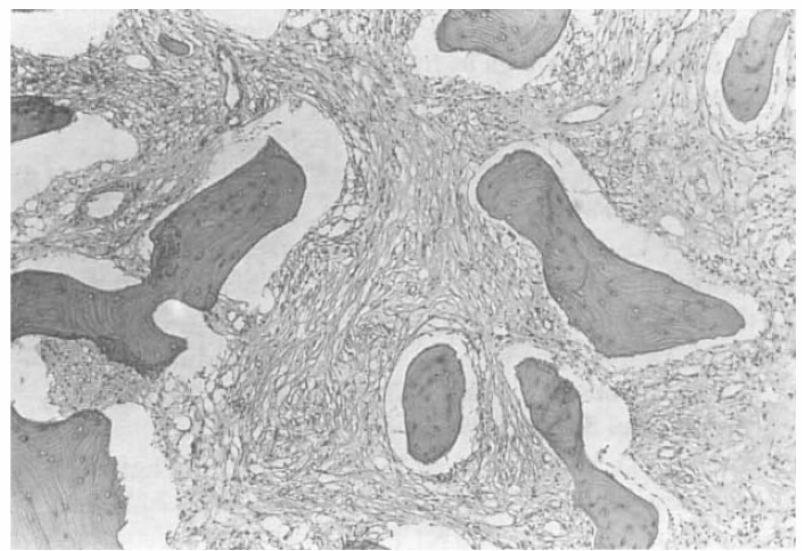

(a)

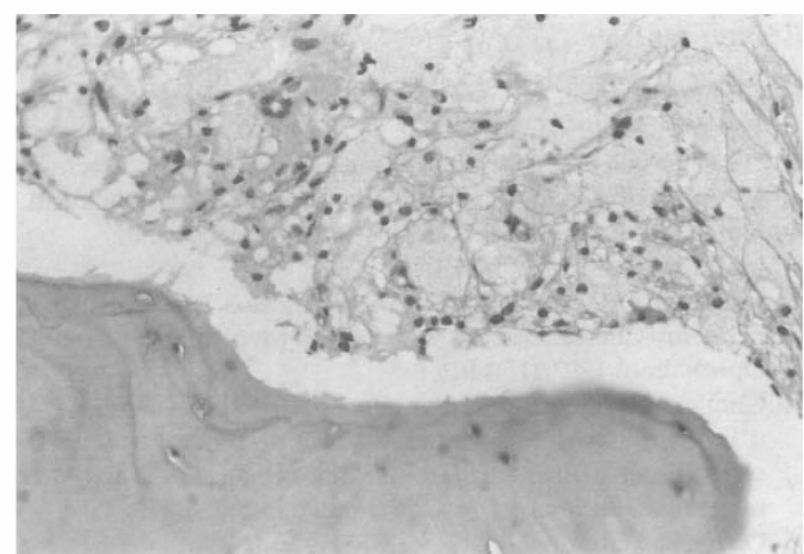

(b)

Fig. 6. Case 2. Tibial biopsy showing sclerosis of marrow (a) and the presence of foamy macrophages in the intertrabecular fibrous tissue (b). Haematoxylin and eosin; (a) $\times 100,(b) \times 400$.

first described by Alper et al. ${ }^{9}$ in 1983 and was reported in 16 of the cases reviewed by Veyssier-Belot et al. ${ }^{2}$ Other clinical features that occur sporadically in these case reports include exophthalmos (16/59), diabetes insipidus $(17 / 59)$, cutaneous xanthomata, usually periocular (11/ $59)$, and retroperitoneal involvement $(17 / 59)$.

The only specific signs for ECD are the radiological and histological findings. Skeletal radiographs show bilateral and symmetrical cortical osteosclerosis of the diaphyseal and metaphyseal regions of long bones. Although pathological examination of tissue is crucial for the diagnosis, it is rarely precise enough, as in our cases, to ensure the diagnosis. In fact, only 11 of the 59 reported cases $^{2}$ had full histological criteria for ECD, which include xanthomatous or xanthogranulomatous infiltrates with fibrosis, and no evidence of Langerhans cell histiocytosis (LCH). Commonly, the pathological report describes 'lipid-laden' macrophages or a xanthogranulomatous infiltration by nests of foamy histiocytes in fibrous tissue, as was seen in our cases. Sheets of xanthoma cells and occasionally Touton giant cells are features which distinguish ECD from sclerosing inflammatory pseudotumour. The absence of expression of S-100 protein and Birbeck granules may be of help to differentiate this condition from $\mathrm{LCH}$; in the cases reported here, the histopathological findings were not consistent with a diagnosis of LCH.

Our cases, the 24th and 25th with orbital involvement, show the consultation of systemic findings that can be seen in ECD and highlight the variability in presentation. Case 1 presented with diabetes insipidus and cardiac involvement prior to the development of exophthalmos, whereas case 2 presented with exophthalmos. Case 1 is unique in that the patient suffered sudden, bilateral and complete loss of vision despite aggressive treatment with radiation, chemotherapy and steroids. The ophthalmological examination at the time of visual loss was not significantly different from an examination 3 days earlier, except for the dramatic change in acuity and the afferent pupillary defect. For this reason, we suspect that the underlying aetiology of the visual loss is a toxic injury to the optic nerve related to the chemotherapy with 2-CDA (cladribine). This may have predisposed the nerves to ischaemic injury when subjected to displacement by the retrobulbar masses.

Cladribine is a purine analogue used for treatment of a broad spectrum of indolent lymphoid malignancies. This agent, and others in its class, are known to be neurotoxic ${ }^{10}$ and a previous case of transient blindness occurring during therapy with cladribine has been reported. ${ }^{11}$ Other forms of chemotherapy have been used in 8 other cases of ECD, with beneficial effects in only 4 cases. Our case 1 is the first reported use of 2-CDA in ECD.

Case 2 presented with bilateral orbital pseudotumours and required long-term azathioprine and two courses of radiotherapy for control of the orbital process. Radiation therapy was utilised in 6 of the patients previously reported without significant effect on the orbital lesions. In our case the response on both occasions to irradiation of the orbit was beneficial whereas only partial control of the retroperitoneal process was achieved by azathioprine. Radiotherapy would appear to have been more important than azathioprine in stabilising the orbital condition. Unfortunately, the development of radiation retinopathy with macular thickening has resulted in a substantial loss of vision in the patient's left eye and required both focal and panretinal photocoagulation in the right. No evidence exists in the literature for intraocular ECD and the appearance clinically and on intravenous fluorescein angiography supported the diagnosis of radiation-induced retinopathy. Furthermore, although retroperitoneal involvement is rarely symptomatic, our patient has had significant problems from his retroperitoneal mass and these continue to be a therapeutic challenge.

In summary, although most patients with ECD do not develop orbital disease, the combination of bilateral xanthelasmata and bilateral, diffuse orbital masses must suggest to the clinician the possibility of ECD, with its serious systemic problems. Implementation of steroid therapy in conjunction with systemic chemotherapy and orbital radiation therapy may have some influence on this otherwise progressive and sight-threatening disease. 


\section{References}

1. Chester W. Über Lipoidgranulomatose. Virchows Arch Pathol Anat 1930;279:561-602.

2. Veyssier-Belot C, Cacoub P, Caparros-Lefebvre D, Wechsler J, Brun B, Remy M, et al. Erdheim-Chester disease: clinical and radiologic characteristics of 59 cases. Medicine (Baltimore) 1996;75:157-69.

3. Valmaggia C, Neuweiler J, Fretz C, Gottlob I. A case of Erdheim-Chester disease with orbital involvement. Arch Ophthalmol 1997;115:1467-8.

4. Chollet P, Eyremandi R, Lesueur L, Arne JL. La maladie $\mathrm{d}^{\prime}$ Erdheim-Chester: une étiologie rare de tumeur retrobulbaire. [Erdheim-Chester disease: a rare etiology of retrobulbar tumour.] J Fr Ophtalmol 1994;17:200-3.

5. Offret H, Hannouche D, Frau E, Doyon D, Quillard J, Schaison G. Maladie d'Erdheim-Chester orbitaire. [Orbital Erdheim-Chester disease]. J Fr Ophtalmol 1995;18:220-5.
6. Bancroft LW, Berquist TH. Erdheim-Chester disease: radiographic findings in five patients. Skeletal Radiol 1998;27:127-32.

7. Wright RA, Hermann RC, Parisi JE. Neurological manifestations of Erdheim-Chester disease. J Neurol Neurosurg Psychiatry 1999;66:72-5.

8. van der Lee I, Slee PH, Elbers JR. A patient with diabetes insipidus and periorbital swellings: Erdheim-Chester disease. Neth J Med 1999;55:76-9.

9. Alper MG, Zimmerman LE, Lapiana FG. Orbital manifestations of Erdheim-Chester disease. Trans Am Ophthalmol Soc 1983;891:64-85.

10. Cheson BD, Vena DA, Foss FM, Sorenson JM. Neurotoxicity of purine analog: a review. J Clin Oncol 1994;12:2216-28.

11. Kobayashi K, Vogelzang NJ, O'Brien SM, Schilsky RL, Ratain MJ. A phase 1 study of intermittent infusion of cladribine in patients with solid tumours. Cancer 1994;74:168-73. 